\title{
Complete genome sequence of Kribbella flavida type strain (IFO 14399')
}

\author{
Rüdiger Pukall ${ }^{1}$, Alla Lapidus ${ }^{2}$, Tijana Glavina Del Rio $^{2}$, Alex Copeland ${ }^{2}$, Hope Tice ${ }^{2}$, Jan- \\ Fang Cheng ${ }^{2}$, Susan Lucas ${ }^{2}$, Feng Chen², Matt Nolan², Kurt LaButti ${ }^{2}$, Amrita Pati ${ }^{2}$, Natalia \\ Ivanova $^{2}$, Konstantinos Mavromatis ${ }^{2}$, Natalia Mikhailova ${ }^{2}$, Sam Pitluck ${ }^{2}$, David Bruce ${ }^{2,3}$, \\ Lynne Goodwin',3, Miriam Land ${ }^{2,5}$, Loren Hauser ${ }^{2,5}$, Yun-Juan Chang ${ }^{2,5}$, Cynthia D. Jeffries ${ }^{2,5}$, \\ Amy Chen ${ }^{4}$, Krishna Palaniappan ${ }^{4}$, Patrick Chainn,3, Manfred Rohde ${ }^{6}$, Markus Göker ${ }^{1}$, Jim \\ Bristow $^{2}$, Jonathan A. Eisen ${ }^{1,7}$, Victor Markowitz ${ }^{4}$, Philip Hugenholtz ${ }^{2}$, Nikos C. Kyrpides ${ }^{2}$, \\ Hans-Peter Klenk ${ }^{1 *}$, and Thomas Brettin ${ }^{2,3}$ \\ ${ }^{1}$ DSMZ - German Collection of Microorganisms and Cell Cultures GmbH, Braunschweig, \\ Germany \\ ${ }^{2}$ DOE Joint Genome Institute, Walnut Creek, California, USA \\ ${ }^{3}$ Los Alamos National Laboratory, Bioscience Division, Los Alamos, New Mexico, USA \\ ${ }^{4}$ Biological Data Management and Technology Center, Lawrence Berkeley National \\ Laboratory, Berkeley, California, USA \\ ${ }^{5}$ Oak Ridge National Laboratory, Oak Ridge, Tennessee, USA \\ ${ }^{6} \mathrm{HZI}$ - Helmholtz Centre for Infection Research, Braunschweig, Germany \\ ${ }^{7}$ University of California Davis Genome Center, Davis, California, USA \\ *Corresponding author: Hans-Peter Klenk
}

Keywords: Actinobacteria, aerobic, soil, mycelia, LL-diaminopimelic acid, Propionibacterineae, Nocardioidaceae, GEBA

The genus Kribbella consists of 15 species, with Kribbella flavida (Park et al. 1999) as the type
species. The name Kribbella was formed from the acronym of the Korea Research Institute of
Bioscience and Biotechnology, KRIBB. Strains of the various Kribbella species were originally
isolated from soil, potato, alum slate mine, patinas of catacombs or from horse racecourses.
Here we describe the features of K. flavida together with the complete genome sequence and
annotation. In addition to the $5.3 \mathrm{Mbp}$ genome of Nocardioides sp. JS614, this is only the
second completed genome sequence of the family Nocardioidaceae. The 7,579,488 bp long
genome with its 7,086 protein-coding and 60 RNA genes and is part of the Genomic Encyc-
lopedia of Bacteria and Archaea project.

\section{Introduction}

Strain IFO $14399^{\mathrm{T}}(=\mathrm{DSM} 17836=$ KCTC $9580=$ JCM $10339=$ NBRC 14399) is the type strain the species Kribbella flavida, which is the type species of the genus Kribbella. Strain IFO $14399^{\mathrm{T}}$ was originally isolated from soil in China and first described as 'Nocardioides fulvus' by Ruan and Zhang, 1979 [1]. In 1999, the strain was reclassified into the novel genus Kribbella on the basis of comparative chemotaxonomic and 16S rRNA sequence analysis [2]. K. flavida exhibits mycelia on several media used for growing the strain. The mycelium consists of hyphae, which are extensively branched and penetrate into the agar medium.
The hyphae often fragment into rod to coccus-like elements [2]. Here we present a summary classification and a set of features for $K$. flavida IFO $14399^{\mathrm{T}}$, together with the description of the complete genomic sequencing and annotation.

\section{Classification and features}

The type strain IFO $14399^{\mathrm{T}}$ was isolated from soil in China. Genbank contains only one additional 16S rRNA gene sequence with at least $99 \%$ similarity, derived from a strain isolated from scabby potatoes (EU80972). No phylotypes from environmental samples or genomic surveys be directly 
linked to $K$. flavida, indicating rare occurrence of the species in so far screened habitats (October 2009). Figure 1 shows the phylogenetic neighborhood of $K$. flavida IF0 $14399^{\mathrm{T}}$ in a $16 \mathrm{~S}$ rRNA based tree. The sequence of the two $16 \mathrm{~S}$ rRNA genes in the genome of strain $14399^{\mathrm{T}}$ differ by two nucleotides from each other and by up to two nucleotides from the previously published $16 \mathrm{~S}$ rRNA sequence generated from KACC 20258 (AY253863).

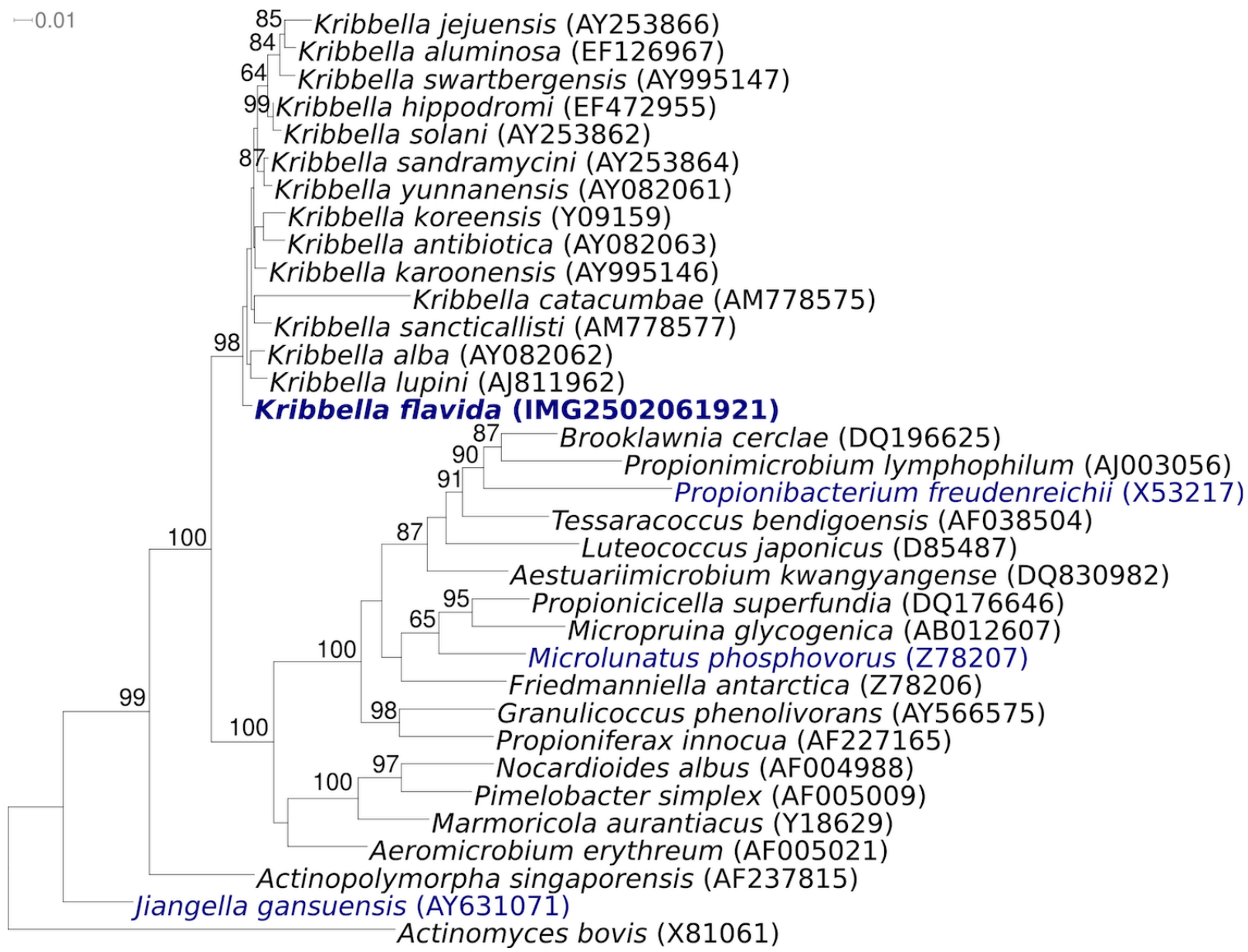

Figure 1. Tree highlighting the position of $K$. flavida IFO $14399^{\top}$ relative to the other type strains of the genus Kribbella and the type strains of the other genera within the families Nocardioidaceae and Propionibacteriaceae. The tree was inferred from 1,343 aligned characters [3,4] of the 16S rRNA gene sequence under the maximum likelihood criterion [5] and rooted in accordance with current taxonomy. The branches are scaled in terms of the expected number of substitutions per site. Numbers above branches are support values from 1,000 bootstrap replicates if larger than $60 \%$. Lineages with type strain genome sequencing projects registered in GOLD [6] are shown in blue, published genomes in bold.

K. flavida is a Gram-positive, aerobic and non-acidfast actinomycete (Table 1), characterized by primary mycelium (Figure 2), with branched hyphae that penetrate into the agar medium. Aerial mycelium is also developed and can break up into short to elongated rod-like elements. Growth occurs between pH 5 and 9 and between 20 and $37^{\circ} \mathrm{C}$. The strain shows positive activity for catalase, oxidase and urease. It utilizes D-glucose, D-cellobiose, maltose, D-melibiose, sucrose, D-trehalose, melezitose, D-raffinose, adonitol, myo-inositol, D- mannitol, inulin, disodium succinate and disodium fumarate as sole carbon and energy source [2].

\section{Chemotaxonomy}

One of the meaningful characteristics of the genus Kribbella is the presence of LL-diaminopimelic acid as the diagnostic diamino acid in the cell wall peptidoglycan [2]. The predominant menaquinone is a tetrahydrogenated menaquinone with nine isoprenoid units $\mathrm{MK}-9\left(\mathrm{H}_{4}\right)$ [2]. The major fatty acids detected in $K$. flavida are anteiso- $\mathrm{C}_{15: 0}$ and 
iso- $\mathrm{C}_{16: 0}$ [2]. Phosphatidylcholine is the main polar lipid [2]. The genus Kribbella differs from other LL-diaminopimelic acid and MK-9(H4) containing taxa, by having a typical hyphal morphology [2].

\section{Genome sequencing and annotation Genome project history}

This organism was selected for sequencing on the basis of its phylogenetic position, and is part of the Genomic Encyclopedia of Bacteria and Archaea project. The genome project is deposited in the Genome OnLine Database [15] and the complete genome sequence is deposited in GenBank. Sequencing, finishing and annotation were performed by the DOE Joint Genome Institute (JGI). A summary of the project information is shown in Table 2.

\section{Growth conditions and DNA isolation}

K. flavida IFO 14399', DSM 17836, was grown in DSM medium 830 [15] at $28^{\circ} \mathrm{C}$. DNA was isolated from 1-1.5 g of cell paste using Qiagen Genomic 500 DNA Kit (Qiagen, Hilden, Germany) following the manufacturer's instructions with modification st/FT for cell lysis according to Wu et al. [16].

\section{Genome sequencing and assembly}

The genome was sequenced using a combination of Sanger and 454 sequencing platforms. All gen- eral aspects of library construction and sequencing can be found on the JGI website. 454 Pyrosequencing reads were assembled using the Newbler assembler version 1.1.01.20 (Roche). Large Newbler contigs were broken into 8,548 overlapping fragments of $1,000 \mathrm{bp}$ and entered into assembly as pseudo-reads. The sequences were assigned quality scores based on Newbler consensus q-scores with modifications to account for overlap redundancy and to adjust inflated q-scores. A hybrid 454/Sanger assembly was made using the parallel phrap assembler (High Performance Software, LLC). Possible mis-assemblies were corrected with Dupfinisher [17] or transposon bombing of bridging clones (Epicentre Biotechnologies, Madison, WI). Gaps between contigs were closed by editing in Consed, custom primer walk or PCR amplification. A total of 2,850 Sanger finishing reads were produced to close gaps, to resolve repetitive regions, and to raise the quality of the finished sequence. Illumina reads were used to improve the final consensus quality using an inhouse developed tool (the Polisher). The error rate of the completed genome sequence is less than 1 in 100,000. Together all sequence types provided $51.2 \times$ coverage of the genome. The final assembly contains 59,008 Sanger and 433,053 pyrosequence reads.

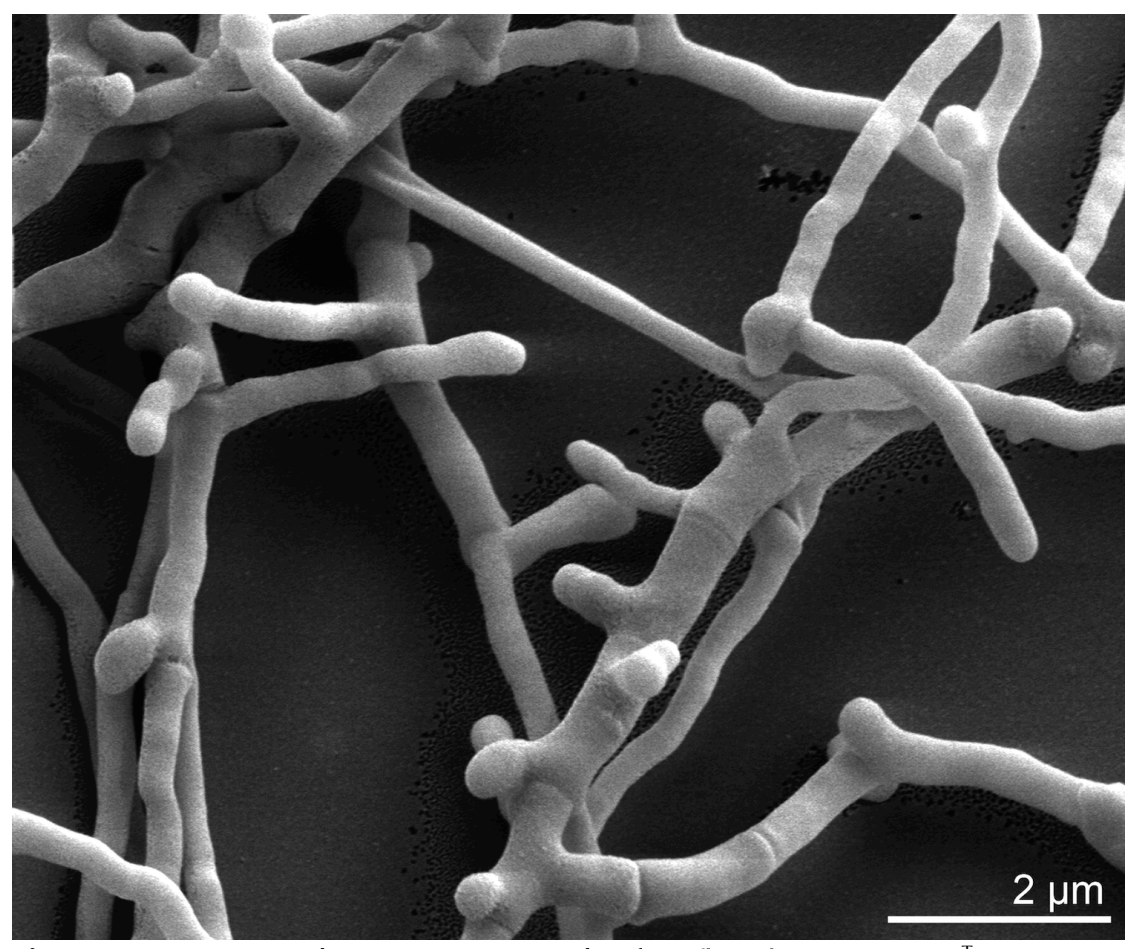

Figure 2. Scanning electron micrograph of K. flavida IFO $14399^{\top}$ 


\begin{tabular}{|c|c|c|c|}
\hline MIGS ID & Property & Term & Evidence code \\
\hline & \multirow{9}{*}{ Current classification } & Domain Bacteria & TAS [8] \\
\hline & & Phylum Actinobacteria & TAS [9] \\
\hline & & Class Actinobacteria & TAS [10] \\
\hline & & Order Actinomycetales & TAS [11] \\
\hline & & Suborder Propionibacterineae & TAS [10] \\
\hline & & Family Nocardioidaceae & TAS [12] \\
\hline & & Genus Kribbella & TAS [2] \\
\hline & & Species Kribbella flavida & TAS [2] \\
\hline & & Type strain IFO 14399 & TAS [2] \\
\hline & Gram stain & positive & TAS [2] \\
\hline & Cell shape & $\begin{array}{l}\text { hyphae, fragmented into rod } \\
\text { to coccoid elements }\end{array}$ & TAS [2] \\
\hline & Motility & nonmotile & NAS \\
\hline & Sporulation & nonsporulating & NAS \\
\hline & Temperature range & $20^{\circ} \mathrm{C}-37^{\circ} \mathrm{C}$ & TAS [2] \\
\hline & Optimum temperature & not reported & \\
\hline & Salinity & not reported & \\
\hline \multirow[t]{3}{*}{ MIGS-22 } & Oxygen requirement & strictly aerobic & TAS [2] \\
\hline & Carbon source & saccharolytic & TAS [2] \\
\hline & Energy source & carbohydrates & TAS [2] \\
\hline MIGS-6 & Habitat & soil & TAS [2] \\
\hline MIGS-15 & Biotic relationship & free living & NAS \\
\hline \multirow[t]{3}{*}{ MIGS-14 } & Pathogenicity & none & NAS \\
\hline & Biosafety level & 1 & TAS [13] \\
\hline & Isolation & soil & TAS $[1,2]$ \\
\hline MIGS-4 & Geographic location & Beijing, China & TAS [1] \\
\hline MIGS-5 & Sample collection time & & NAS \\
\hline MIGS-4.1 & Latitude & 39.55 & NAS \\
\hline MIGS-4.2 & Longitude & 116.25 & בהקה \\
\hline MIGS-4.3 & Depth & not reported & \\
\hline MIGS-4.4 & Altitude & not reported & \\
\hline
\end{tabular}

Evidence codes - IDA: Inferred from Direct Assay (first time in publication); TAS: Traceable Author Statement (i.e., a direct report exists in the literature); NAS: Non-traceable Author Statement (i.e., not directly observed for the living, isolated sample, but based on a generally accepted property for the species, or anecdotal evidence). These evidence codes are from of the Gene Ontology project [14]. If the evidence code is IDA, then the property was directly observed by one of the authors or an expert mentioned in the acknowledgements.

\section{Genome annotation}

Genes were identified using Prodigal [18] as part of the Oak Ridge National Laboratory genome annotation pipeline, followed by a round of manual curation using the JGI GenePRIMP pipeline [19]. The predicted CDSs were translated and used to search the National Center for Biotechnology Information (NCBI) nonredundant database, UniProt, TIGRFam, Pfam, PRIAM, KEGG, COG, and InterPro databases. Additional gene prediction analysis and manual functional annotation was performed within the Integrated Microbial Genomes Expert Review (IMG-ER) platform [20].

\section{Genome properties}

The genome is 7,579,488 bp long with a $70.6 \%$ GC content (Table 3 and Figure 3). Of the 7,146 genes predicted, 7,086 were protein-coding genes, and 60 RNAs; 143 pseudogenes were also identified. The majority of the protein-coding genes (70.7\%) were assigned with a putative function while those remaining were annotated as hypothetical proteins. The distribution of genes into COGs functional categories is summarized in Table 4. 
Table 2. Genome sequencing project information

\begin{tabular}{lll}
\hline MIGS ID & Property & Term \\
\hline MIGS-31 & Finishing quality & $\begin{array}{l}\text { Finished } \\
\text { Two Sanger libraries: 8kb pMCL200 } \\
\text { and fosmid pcc1 Fos } \\
\text { One 454 pyrosequence standard library } \\
\text { and one Standard Illumina library }\end{array}$ \\
MIGS-28 & Libraries used & ABI3730, 454 GS FLX, Illumina GA \\
MIGS-31.2 & Sequencing platforms & S.4× Sanger; 13.4× pyrosequence \\
MIGS-30 & Assemblers & Newbler 1.1.01.20, phrap \\
MIGS-32 & Gene calling method & Prodigal, GenePRIMP \\
& INSDC ID & CP001736 \\
& Genbank Date of Release & January 13, 2010 \\
& GOLD ID & Gc01192 \\
& NCBI project ID & 21089 \\
& Database: IMG-GEBA & 2501939632 \\
MIGS-13 & Source material identifier & DSM 17836 \\
& Project relevance & Tree of Life, GEBA \\
\hline
\end{tabular}

Table 3. Genome Statistics

\begin{tabular}{lrr}
\hline Attribute & Value & \% of Total \\
\hline Genome size (bp) & $7,579,488$ & $100.00 \%$ \\
DNA coding region (bp) & $6,893,122$ & $90.94 \%$ \\
DNA G+C content (bp) & $5,348,686$ & $70.57 \%$ \\
Number of replicons & 1 & \\
Extrachromosomal elements & 0 & \\
Total genes & 7,146 & $100.00 \%$ \\
RNA genes & 60 & $0.84 \%$ \\
rRNA operons & 2 & \\
Protein-coding genes & 7,086 & $99.16 \%$ \\
Pseudo genes & 143 & $2.00 \%$ \\
Genes with function prediction & 5,049 & $70.65 \%$ \\
Genes in paralog clusters & 1,595 & $22.32 \%$ \\
Genes assigned to COGs & 4,877 & $68.25 \%$ \\
Genes assigned Pfam domains & 5,174 & $72.40 \%$ \\
Genes with signal peptides & 1,721 & $24.08 \%$ \\
Genes with transmembrane helices & 1,675 & $23.44 \%$ \\
CRISPR repeats & 0 & 0 \\
\hline
\end{tabular}




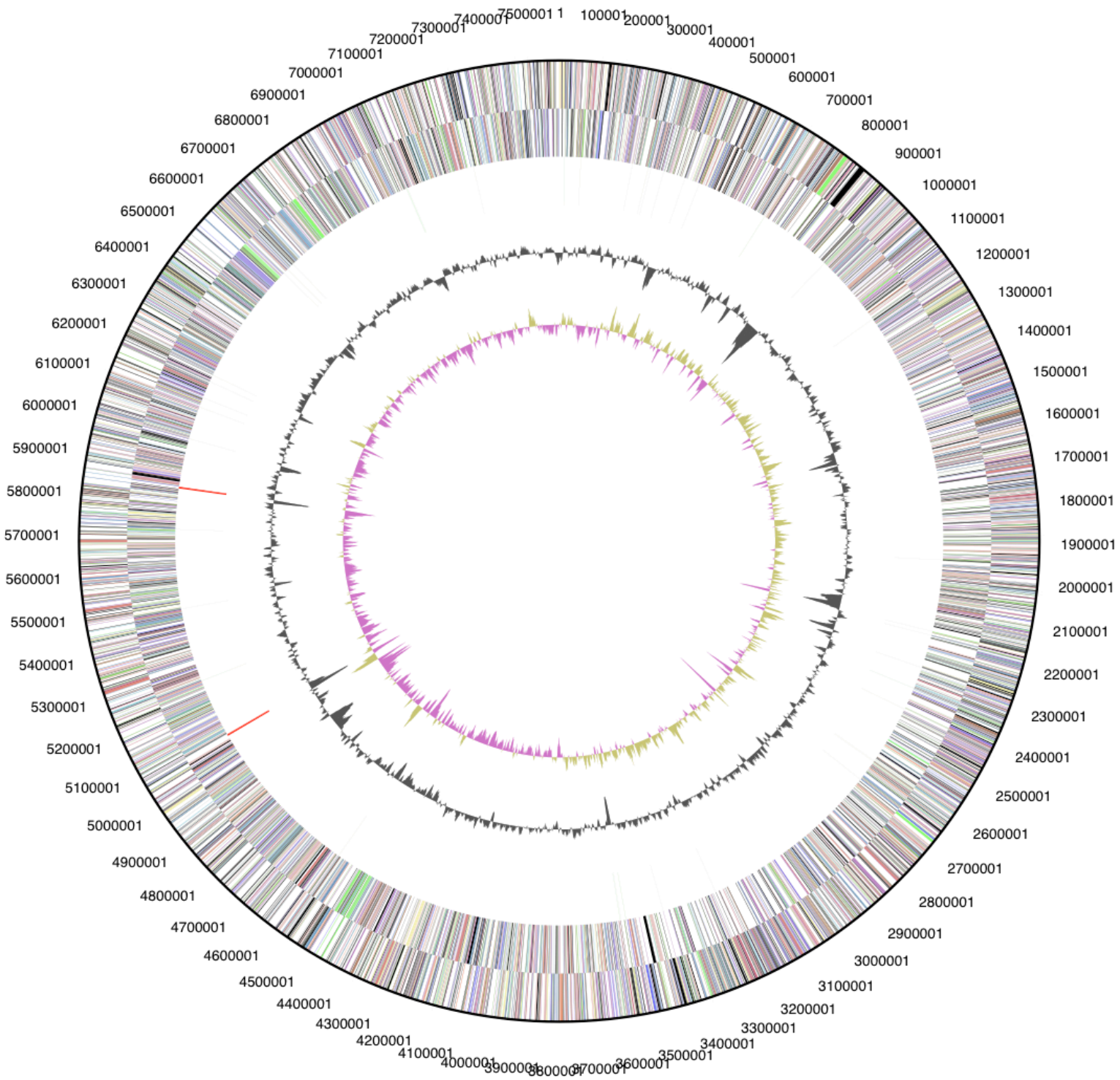

Figure 3. Graphical circular map of the chromosome. From outside to the center: Genes on forward strand (color by COG categories), Genes on reverse strand (color by COG categories), RNA genes (tRNAs green, rRNAs red, other RNAs black), GC content, GC skew.

Table 4. Number of genes associated with the general COG functional categories

\begin{tabular}{crrl} 
Code & Value & \%age & Description \\
\hline J & 225 & 4.1 & Translation, ribosomal structure and biogenesis \\
A & 2 & 0.0 & RNA processing and modification \\
K & 762 & 13.8 & Transcription \\
L & 184 & 3.3 & Replication, recombination and repair \\
B & 1 & 0.0 & Chromatin structure and dynamics \\
D & 38 & 0.7 & Cell cycle control, cell division, chromosome partitioning \\
Y & 0 & 0.0 & Nuclear structure \\
V & 136 & 2.5 & Defense mechanisms \\
T & 261 & 4.7 & Signal transduction mechanisms \\
M & 239 & 4.3 & Cell wall/membrane biogenesis \\
\hline
\end{tabular}


Table 4 (cont.). Number of genes associated with the general COG functional categories

\begin{tabular}{crrl}
\hline Code & Value & \%age & Description \\
\hline N & 2 & 0.0 & Cell motility \\
Z & 2 & 0.0 & Cytoskeleton \\
W & 0 & 0.0 & Extracellular structures \\
U & 46 & 0.8 & Intracellular trafficking and secretion \\
O & 143 & 2.6 & Posttranslational modification, protein turnover, chaperones \\
C & 308 & 5.6 & Energy production and conversion \\
G & 636 & 11.5 & Carbohydrate transport and metabolism \\
E & 397 & 7.2 & Amino acid transport and metabolism \\
F & 100 & 1.9 & Nucleotide transport and metabolism \\
H & 264 & 4.8 & Coenzyme transport and metabolism \\
I & 212 & 3.8 & Lipid transport and metabolism \\
P & 218 & 3.9 & Inorganic ion transport and metabolism \\
Q & 175 & 3.2 & Secondary metabolites biosynthesis, transport and catabolism \\
R & 802 & 14.5 & General function prediction only \\
S & 367 & 6.7 & Function unknown \\
- & 2,269 & 31.8 & Not in COGs \\
\hline
\end{tabular}

\section{Acknowledgements}

We would like to gratefully acknowledge the help of Katja Steenblock for growing $K$. flavida cultures and Susanne Schneider for DNA extraction and quality analysis (both at DSMZ). This work was performed under the auspices of the US Department of Energy's Office of Science, Biological and Environmental Research Program, and by the University of California, Lawrence Berkeley National Laboratory under contract No. DE-

\section{References}

1. Ruan J, Zhang Y. Two new species of Nocardioides. Acta Microbiol Sin 1979; 19:347-352.

2. Park YH, Yoon JH, Shin YK, Suzuki K, Kudo T, Seino A, Kim HJ, Lee JS, Lee ST. Classification of 'Nocardioides fulvus' IFO 14399 and Nocardioides sp. ATCC 39419 in Kribbella gen. nov., as Kribbella flavida sp. nov. and Kribbella sandramycini sp. nov. Int / Syst Bacteriol 1999; 49:743-752. PubMed doi:10.1099/00207713-49-2-743

3. Castresana J. Selection of conserved blocks from multiple alignments for their use in phylogenetic analysis. Mol Biol Evol 2000; 17:540-552. PubMed

4. Lee C, Grasso C, Sharlow MF. Multiple sequence alignment using partial order graphs. Bioinformatics 2002; 18:452-464. PubMed doi:10.1093/bioinformatics/18.3.452
AC02-05CH11231, Lawrence Livermore National Laboratory under Contract No. DE-AC52-07NA27344, Los Alamos National Laboratory under contract No. DEAC02-06NA25396, and Oak Ridge National Laboratory under contract DE-AC05-000R22725, as well as German Research Foundation (DFG) INST 599/1-1 and SI 1352/1-2.

5. Stamatakis A, Hoover P, Rougemont J. A Rapid Bootstrap Algorithm for the RAxML Web Servers. Syst Biol 2008; 57:758-771. PubMed doi:10.1080/10635150802429642

6. Liolios K, Chen IM, Mavromatis K, Tavernarakis N, Hugenholtz P, Markowitz VM, Kyrpides NC. The Genomes On Line Database (GOLD) in 2009: status of genomic and metagenomic projects and their associated metadata. Nucleic Acids Res 2010; 38:D346-D354. PubMed doi:10.1093/nar/gkp848

7. Field D, Garrity G, Gray T, Morrison N, Selengut J, Sterk P, Tatusova T, Thomson N, Allen MJ, Angiuoli SV, et al. The minimum information about a genome sequence (MIGS) specification. Nat Biotechnol 2008; 26:541-547. PubMed doi:10.1038/nbt1360 
8. Woese CR, Kandler O, Wheelis ML. Towards a natural system of organisms: proposal for the domains Archaea, Bacteria, and Eucarya. Proc Natl Acad Sci USA 1990; 87:4576-4579. PubMed doi:10.1073/pnas.87.12.4576

9. Garrity GM, Holt JG. The Road Map to the Manual. In: Garrity GM, Boone DR, Castenholz RW (eds), Bergey's Manual of Systematic Bacteriology, Second Edition, Springer, New York, 2001, p. 119-169.

10. Stackebrandt E, Rainey FA, Ward-Rainey NL. Proposal for a new hierarchic classification system, Actinobacteria classis nov. Int J Syst Bacteriol 1997; 47:479-491. doi:10.1099/00207713-47-2$\underline{479}$

11. Skerman VBD, McGowan V, Sneath PHA. Approved Lists of Bacterial Names. Int J Syst Bacteriol 1980; 30:225-420. doi:10.1099/0020771330-1-225

12. Nesterenko OA, Kvasnikov El, Nogina TM. Nocardioidaceae fam. nov., a new family of the order Actinomycetales Buchanan 1917. Mikrobiol Zh 1985; 47:3-12.

13. Classification of Bacteria and Archaea in risk groups www.baua.de TRBA 466.

14. Ashburner M, Ball CA, Blake JA, Botstein D, Butler H, Cherry JM, Davis AP, Dolinski K, Dwight SS, Eppig JT, et al. Gene Ontology: tool for the unification of biology. Nat Genet 2000; 25:25-29. $\underline{\text { PubMed doi:10.1038/75556 }}$
15. List of growth media used at DSMZ: http://www.dsmz.de/microorganisms/media_list.p $\underline{\mathrm{hp}}$

16. Wu D, Hugenholtz P, Mavromatis K, Pukall R, Dalin E, Ivanova N, Kunin V, Goodwin L, Wu M, Tindall BJ, et al. A phylogeny-driven genomic encyclopedia of Bacteria and Archaea. Nature 2009; 462:1056-1060. PubMed doi:10.1038/nature08656

17. Sims D, Brettin T, Detter JC, Han C, Lapidus A, Copeland A, Glavina Del Rio T, Nolan M, Chen F, Lucas S, et al. Complete genome of Kytococcus sedentarius type strain $\left(541^{\mathrm{T}}\right)$. Stand Genomic Sci 2009; 1:12-20. doi:10.4056/sigs.761

18. Hyatt D, Chen GL, Locascio PF, Land ML, Larimer FW, Hauser LJ. Prodigal Prokaryotic Dynamic Programming Genefinding Algorithm. BMC Bioinformatics 2010; 11:119. PubMed doi:10.1186/1471-2105-11-119

19. Pati A, Ivanova N, Mikhailova, N, Ovchinikova G, Hooper SD, Lykidis A, Kyrpides NC. GenePRIMP: A Gene Prediction Improvement Pipeline for microbial genomes. Nat Methods (in press)

20. Markowitz VM, Mavromatis K, Ivanova NN, Chen IMA, Chu K, Kyrpides NC. IMG ER: a system for microbial genome annotation expert review and curation. Bioinformatics 2009; 25:2271-2278. $\underline{\text { PubMed doi:10.1093/bioinformatics/btp393 }}$ 